\title{
Attitude Estimation Based on Modified Two-stage EKF
}

\author{
Peinan Wang, Zhiqiang Wang and Qing Zhu \\ Beijing University of Technology, Beijing, China
}

\begin{abstract}
In this paper, an attitude estimation algorithm based on modified two-stage Extended Kalman Filter (EKF) is presented to improve the accuracy of attitude estimation based on Micro Electro-Mechanical System (MEMS) motion capture technology. Firstly, a two-stage EKF is presented to replace the conventional EKF, to reduce the computational complexity and computing time. Secondly, the normalized quaternion model is applied instead of Euler Angle model to reduce the calculation error of attitude estimation. Finally, the automatic error compensation realized through constructing the acceleration error covariance operator to adjust error covariance matrix according to the measurement values of the acceleration. The experiment result shows that the proposed method can significantly reduce the computational complexity and the size of universal joint deadlocks and linear acceleration effect on the attitude estimation.
\end{abstract}

Keywords-extended kalman filter; motion capture; attitude estimation

\section{INTRODUCTION}

Human motion capture is a technology for measuring the human body movement information in three-dimensional space by using digital information technology, which has been widely used in sports, games, bio-mechanics, virtual reality, and other areas of the human-computer interface. The commonly used motion capture technology in principle can be divided into the following four kinds, optical motion capture technology, acoustic motion capture technology, electromagnetic motion capture technology and Micro ElectroMechanical System (MEMS) motion capture technology. In recent years, with the development of the MEMS technology, MEMS motion capture has become an important part of human motion capture system [1]. MEMS is able to capture human motion and attitude though the inertial measurement unit device. It mainly contains three axis gyroscope and threeaxis accelerometer. However, as for the noise and drift error of the inertial measurement sensor will accumulate, it makes the precision of attitude estimation become difficult [2, 3].

To get the accurate attitude estimation, many algorithms are proposed such as complementary filter and Kalman filter. Complementary filter has the advantages of low computational complexity and easy to implement, but the precision of attitude estimation is not as accurate as Kalman filter. Li-jie Zhang combined the information from gyroscope, accelerometer and magnetometer using Kalman filter algorithm, which improves the precision of the attitude estimation [4]. To further improve the performance of the Kalman filter, Marins proposed an Extended Kalman Filter
(EKF) method [5]. The method used quaternion model to describe the attitude, which avoided the singularity problem of attitude estimation, and improved the practicability. But the computational complexity is quite large, the linearization of the non-linear system may lead to lead to instability, the method should be used under the assumption of white noise, which restrict the application of the method. For this reason, we propose a new attitude estimation method using two-stage EKF and automatic error compensation to improve the accuracy of attitude estimation for motion capture.

\section{AtTITUde Estimation ModeLing}

\section{A. Attitude Description and Motion Equation}

The section headings are in boldface capital and lowercase letters. Second level headings are typed as part of the succeeding paragraph (like the subsection heading of this paragraph).

Generally, in the study of human body movement, the geographic coordinate system (NEU coordinate system) is a constant, choose the attitude estimation system in inertial coordinate system [6]. The space motion in NEU coordinate system could be supposed as a rotating composition result from the inertial coordinate system. As shown in Figure 1, $\theta$ is the pitch angle, $\phi$ is the roll angle and $\psi$ is the yaw angle.

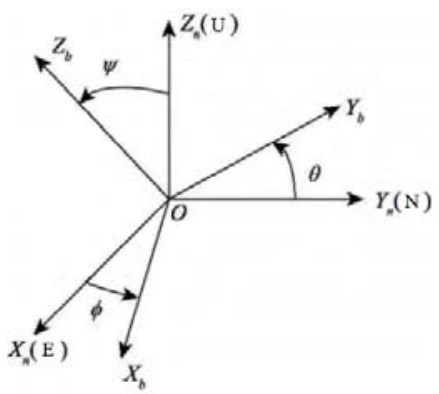

FIGURE I. THE TRANSITION RELATIONSHIP OF ANGLE BETWEEN NEU AND INERTIAL COORDINATE SYSTEMS

The data from the sensors is based on the inertial coordinate system, so the attitude estimation needs to convert the inertial coordinate system to the NEU coordinates system, and the rotation matrix ${ }^{S}{ }_{b}^{n}$ can be expressed as: 
$S_{b}^{n}=\left[\begin{array}{ccc}\cos \theta \cos \psi & -\cos \phi \sin \psi+\sin \phi \sin \theta \cos \psi & \sin \phi \sin \psi+\cos \phi \sin \theta \cos \psi \\ \cos \theta \sin \psi & \cos \phi \cos \psi+\sin \phi \sin \theta \sin \psi & -\sin \phi \cos \psi+\cos \phi \sin \theta \sin \psi \\ -\sin \theta & \sin \phi \cos \theta & \cos \phi \cos \theta\end{array}\right]$

In the attitude fusion process, quaternion is applied to describe the body's attitude, which means the data from the sensors are converted to quaternion. Consider $Q$ as a quaternion vector, which is:

$$
Q=q_{0}+q_{1} \vec{i}+q_{2} \overrightarrow{j i}+q_{3} \overrightarrow{k i}
$$

Where $q_{0}$ is the scalar part of the quaternion and $\overrightarrow{q_{0}}=\left[q_{2}, q_{3}, q_{4}\right]^{T}$ is the vector part of the quaternion.

For the attitude estimation, the quaternion is normalized in each filtering cycle as below

$$
Q=\frac{Q}{\sqrt{q_{0}^{2}+q_{1}^{2}+q_{2}^{2}+q_{3}^{2}}}
$$

As both NEU and inertial coordinate systems are based on rectangular coordinate system, the coordinate system can be supposed as a rigid body. The relationship of the space angle between the two coordinate systems can be considered as the fixed-point motion of rigid body. Therefore, in combination with quaternion, rotation matrix between the two coordinate systems $S_{b}^{n}$ can be expressed as

$$
S_{b}^{n}=\left[\begin{array}{lll}
1-2\left(q_{2}^{2}+q_{3}^{2}\right) & 2\left(q_{1} q_{2}-q_{0} q_{3}\right) & 2\left(q_{1} q_{3}+q_{0} q_{2}\right) \\
2\left(q_{1} q_{2}+q_{0} q_{3}\right) & 1-2\left(q_{1}^{2}+q_{3}^{2}\right) & 2\left(q_{2} q_{3}-q_{0} q_{1}\right) \\
2\left(q_{1} q_{3}-q_{0} q_{2}\right) & 2\left(q_{2} q_{3}+q_{0} q_{1}\right) & 1-2\left(q_{1}^{2}+q_{2}^{2}\right)
\end{array}\right]
$$

Then the attitude angles can be described as follow

$$
\begin{gathered}
\theta=\arcsin \left(2\left(q_{0} q_{2}-q_{1} q_{3}\right)\right) \\
\phi=\arctan \frac{2\left(q_{2} q_{3}+q_{0} q_{1}\right)}{1-2\left(q_{1}^{2}+q_{2}^{2}\right)} \\
\psi=\arctan \frac{2\left(q_{1} q_{2}+q_{0} q_{3}\right)}{1-2\left(q_{2}^{2}+q_{3}^{2}\right)}
\end{gathered}
$$

\section{B. Sensor Modeling}

The error of gyroscope is consisting of random drift and noise. To analyze its characteristics preferably, the model of gyroscope is established as below

$$
\omega_{m}=\omega_{t}+b_{0}+\omega_{a}+b
$$

where $\omega_{m}$ indicates the measuring angular velocity, $\omega_{t}$ indicates the real angular velocity, $b_{0}$ indicates the constant drift, $b$ indicates the time-varying drift, $\omega_{a}$ indicates the noise which can be considered as white noise.

In the same way, the accelerometer model can be established as follow

$$
a_{m}=a_{b}-g^{b}+v_{a}
$$

where ${ }^{a_{m}}$ indicates the measuring value of accelerometer, $a_{b}$ indicates the real value of accelerated velocity, $g^{b}$ indicates the projection of gravitational acceleration projecting on the body, $v_{a}$ indicates the white noise. As for the gravitational field in NEU coordinate system can be presented as $g^{n}=[0,0, g]^{T}$, therefore, $g^{b}=S_{n}^{b} g^{n}$.

\section{Modified AtTitude Estimation}

\section{A. Kalman Filter}

Kalman filter is an efficient auto-regression filter which was proposed by R.E.Kalman to solve filter problem for discrete linear system. It introduced the concept of state space into filter technique. The estimated signal is considered as the state and the state equation is used to describe system. Therefore, Kalman filter can be able to solve the estimation problem of multidimensional non-stationary random process which was difficult to solve before. With the rapid development of digital computing technology, the Kalman filter has been widely researched and applied.

Kalman filter is defined as: in a linear system, a $n \times 1$ vector $\vec{X}$ follows the discrete time evolution as below

$$
\vec{X}_{k+1}=A \vec{X}_{k}+B \vec{u}_{k}+\vec{\omega}_{k}
$$

where $A$ indicates a $n \times n$ state-transition matrix, $B$ indicates a $n \times p$ state-input matrix, $\vec{\omega}_{k}$ indicates process noise. Suppose there is $m$ direct system measurement vector as

$$
\vec{y}_{k}=C \vec{x}_{k}+\vec{v}_{k}
$$

where $C$ indicates the $m \times n$ state fusion matrix, and the Kalman filter is defined as below

$$
K_{k}=A P_{k} C^{T}\left(C P_{k} C^{T}+R_{k}\right)^{-1}
$$




$$
\begin{gathered}
\vec{x}_{k+1}=\left(A \vec{x}_{k}+B \vec{u}_{k}\right)+K_{k}\left(\vec{y}_{k}-C \vec{x}_{k}\right) \\
P_{k+1}=A P_{k} A^{T}+Q_{k}+A P_{k} C^{T} R_{k}^{-1} C P_{k} A^{T}
\end{gathered}
$$

where $Q_{k}$ indicates the uncertainty covariance matrix, $K$ indicates Kalman gain matrix, $C_{b}^{n}$ is the error estimation.

Kalman filter can estimate the state toward the real value from the measured process with noise, which only needs the former state data for each calculation. This character is suitable for real-time processing and computing.

\section{B. Modified EKF Method}

As the gyroscope will produce drift in motion, but the accelerometer and magnetometer won't produce time drift. So we can use of the accelerometer and magnetometer to compensate for the lack of gyroscope in the process of dynamic measurement. Therefore, in attitude estimation based on Kalman filtering, accelerometer and magnetometer data is often used as the observation variable to do the auxiliary correction, and the results of calculated gyro are based on EKF, and adopt gauss-newton iteration method for iteration to find is the most appropriate correction.

Commonly used nine axis attitude estimation method based on Kalman filter generally use single EKF. The threeaxis accelerometer and three-axis magnetometer are used to establish 6-dimension observation variables, which become to the $6 \times 4$ observation matrix in EKF. This process may produce great amount of computational complexity. In one iteration of EKF, a total of 857 times multiplication and 663 times addition are needed.

For the two-stage EKF, in the first filter, we use the accelerometer to correct the roll angle and pitch angle, in which an $3 \times 4$ observation matrix is established. In the second filter, we use the magnetometer to correct yaw angle, in which an $3 \times 4$ observation matrix is established. The two-stage EKF updates attitude estimation in one iteration. It needs a total of 652 times multiplication and 576 times addition. Therefore, the two-stage EKF can reduce the computing time, shorten the operation time and improve the efficiency of attitude estimation compared with single EKF.

According to the analysis above, the two-stage EKF is applied instead of the more commonly used single EKF in this paper. An adaptive error covariance algorithm is involved in the first stage EKF to correct the roll angle and pitch angle. In the second stage EKF, the yaw angle is corrected through the magnetic meter. The specific method is as follows:

Quaternion is used as attitude state. According to the gyroscope data, system state equations can be established as follow

$$
q_{(k+1)}=A_{(k+1)} q_{(k)}+B_{(k+1)} V_{(k+1)}
$$

Establish the observation equation of the first stage EKF according to the accelerometer as follows

$$
a_{(k+1)}=H_{1} q_{(k+1)}+b_{(k+1)}+W_{1(k+1)}
$$
below

Calculate the prior error variance of the first stage EKF as

$$
P_{(k+1)}=A_{(k+1)} P_{(k)} A_{(k+1)}^{T}+Q_{(k+1)}
$$

Generally, commonly used 9-axis attitude estimation based on Kalman filter is not able to eliminate the error from the linear motion acceleration. In this paper, an adaptive acceleration error covariance operator is constructed as below

$$
R=k_{a}\left(\left\|a_{k}\right\|-\|g\|\right) I_{3 \times 3}+R_{\text {Noise }}
$$

Where $k_{a}$ is weighted factor, $R$ is acceleration error convariance matrix, $a_{k}$ is accelerated velocity measured by accelerometer, $g$ is gravitational acceleration, $R_{\text {Noise is the }}$ noise covariance.

Kalman filtering system update process is mainly completed through the following two equations:

$$
\begin{gathered}
q_{(k+1)}=q_{(k)}+K\left(a_{c}-H_{q_{(k)}}\right) \\
K=P^{-} H^{T}\left(H P^{-} H^{T}+R\right)^{-1}
\end{gathered}
$$

Where $K$ indicates Kalman gain matrix, $P^{-}$indicates prior error covariance, $H$ indicates the quaternion Jacobian matrix of acceleration, $q_{(k)}$ indicates the attitude quaternion at $k$ moment.

According to Eq. 17 and Eq. 18, it shows that the Kalman gain matrix $K$ have the effect of modified the original attitude quaternion, and the regulating range of $K$ is correction of the quaternion and the related with $R$. In this paper, by constructing the acceleration error covariance operator $R$, the error covariance matrix can be regulated according to the measured value of the acceleration. By this way, automatic error compensation is realized. On one hand, when there is no linear motion acceleration, the acceleration to measure acceleration should be corresponding adaptive acceleration error covariance matrix $R$ which is close to noise measurement error covariance. On the other hand, when the linear acceleration increases, the element value of the error covariance matrix also can become big. By adjusting $R$, the 
element value of the Kalman gain matrix become smaller. As can be seen from the Eq. 17, the elements in the matrix value decreased accordingly, thus the quantity of state quaternion error is reduced.

The Kalman filter gain in the first stage of EKF is expressed as below

$$
K_{1(k+1)}=P_{(k+1)} H_{1(k+1)}^{T}\left(H_{1(k+1)} P_{(k+1)} H_{1(k+1)}^{T}+R_{1(k+1)}\right)^{-1}
$$

where $H_{1}$ is the system observation matrix. Update the status value of the quaternion (correct roll angle and pitch angle only)

$$
q_{(k+1)}=q_{(k+1)}+K_{1(k+1)}\left(a_{k+1}-H_{1(k+1)} q_{(k)}\right)
$$

Due to the roll angle and pitch angle corresponding to the quaternion $q_{0} 、 q_{1} 、 q_{2}$ of $q$, in the first stage EKF, we only need to modify the former three elements, so there is no change for $q_{3}$. That means:

$$
q_{(k+1)}=q_{(k+1)}+\left(q_{0}, q_{1}, q_{2}, 0\right)
$$

Establish the observation equation of the second stage EKF according to the magnetometer as

$$
m_{(k+1)}=H_{2} q_{(k+1)}+W_{2(k+1)}
$$

where the state vector $q_{(k+1)}$ indicates the rotation quaternion at $k+1$ moment, $m_{(k+1)}$ is the magnetometer measurement vector at $k+1$ moment, $H_{2}$ is the observation matrix of magnetometer, $V_{(k+1)}, W_{1(k+1)}, W_{2(k+1)}$ are gyroscope, accelerometer , magnetometer measurement noise matrix at $k+1$ moment, the measurement noise can be described as a Gaussian white noise (zero mean variance, respectively $\sigma_{\omega}^{2} I$, $\left.\sigma_{a}^{2} I, \sigma_{m}^{2} I\right)$.

Calculate the prior error variance of the second stage EKF as below

$$
P_{(k+1)}=\left(I-K_{1(k+1)} H_{1(k+1)}\right) P_{(k+1)}
$$

Calculate the Kalman gain of the second stage EKF as below

$$
K_{2(k+1)}=P_{(k+1)} H_{2(k+1)}^{T}\left(H_{2(k+1)} P_{(k+1)} H_{2(k+1)}^{T}+R_{2(k+1)}\right)^{-1}
$$

Update the status value of the quaternion (correct yaw angle only)

$$
q_{(k+1)}=q_{(k)}+K_{2(k+1)}\left(m_{(m+1)}-H_{2(k+1)} q_{(k)}\right)
$$

The quaternion is corrected in the second stage EKF, which only yaw angle is corrected. The yaw angle corresponds to $q_{0}$ and $q_{3}$, which we should only change the two values. The other values remain the same, which is equivalent to:

$$
q_{(k+1)}=q_{(k)}+\left(q_{0}, 0,0, q_{3}\right)
$$

Calculate the posteriori error variance as follow

$$
P_{(k+1)}=\left(I-K_{1(k+1)} H_{2(k+1)}\right) P_{(k+1)}
$$

Iterate the process of the above, and then use Eq. 5 to estimate the attitude angle. The flow chart of proposed method is shown in figure 2 .

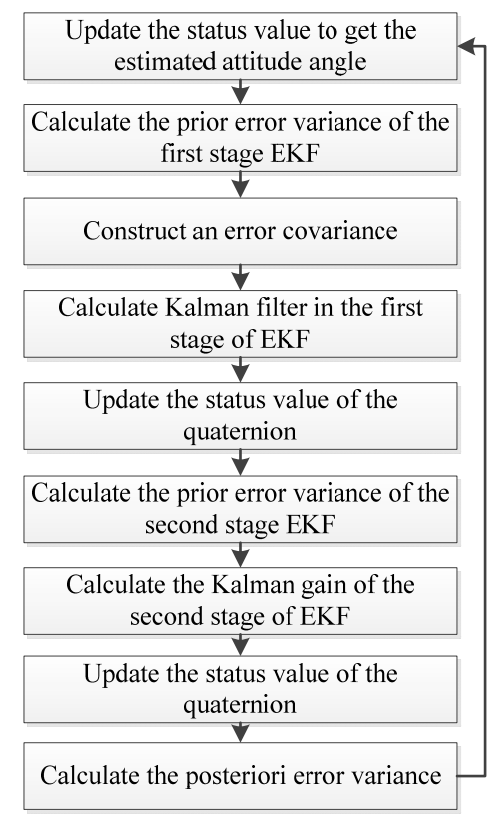

FIGURE II. FLOW CHART OF PROPOSED METHOD

\section{EXPERIMENT RESULTS}

Through the experiment of the collected data, we compared the error from proposed method with the error from the method of Animazoo IGS, and the results are as follows. 

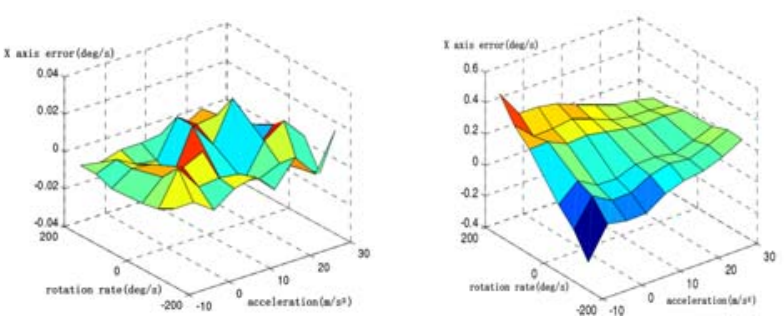

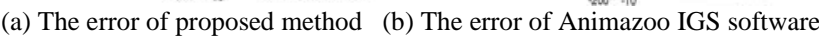
FIGURE III. X AXIS OUTPUT ERROR
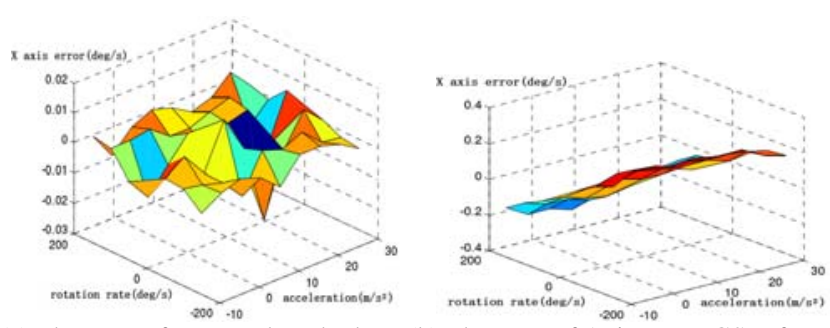

(a) The error of proposed method

(b) The error of Animazoo IGS software

FIGURE IV. Y AXIS OUTPUT ERROR
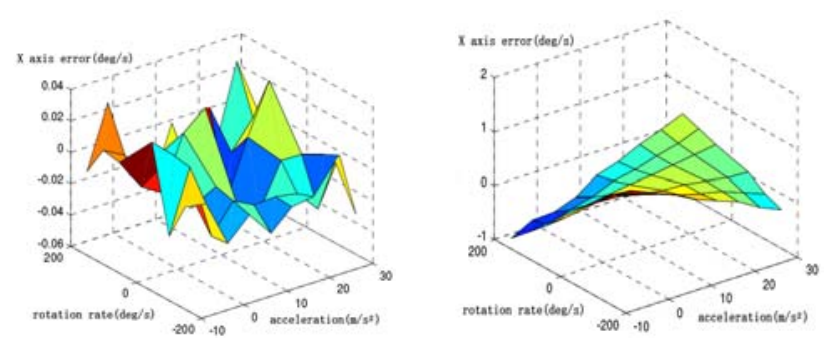

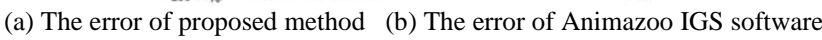
FIGURE V. Z AXIS OUTPUT ERROR

As shown in figure 3, figure 4 and figure 5, the error of attitude estimation using the proposed method in terms of $X$, $\mathrm{Y}, \mathrm{Z}$ three axes is between 0.06 0.04. Compared with the Animazoo IGS, its error is decreased significantly, thus it also proved that the proposed method is more accurate.

\section{CONCLUSION}

In this paper, a modified attitude estimation method based on two-stage EKF is presented. Firstly, the main deficiencies of the existing research results are introduced. Secondly, an improved design for the above problems based on the twostage EKF is proposed. By using the error covariance operator with adaptive compensation function to eliminate the error due to the linear acceleration motion. Finally, through the rigorous experimental comparison and analysis, the effectiveness of the proposed method is proved.

\section{REFERENCES}

[1] XIANG Ze-rui, ZHI Jin-yi, XU Bo-chu, LI Juan. Survey on motion capture technique and its applications [J]. Application Research of Computers,2013,20(8):2241-2245

[2] WANG Ke-jun, CHEN We, Design and implementation of wearable human motion capture system [J]. Journal of Engineering of Heilongjiang University, 2013, 4(2): 97-102.
[3] K.Adistambha, C.H.Ritz, I.S.Burnett, Motion classification using Dynamic Time Warping. 2008 IEEE 10th Workshop on Multimedia Signal Processing,2008:622 627.

[4] S.Sempena, D. N.U.Maulidevi, S.T, M.Sc, et al., Human Action Recognition Using Dynamic Time Warping. International Conference on Electrical Engineering and Informatics (ICEEI), 2011:1 5

[5] Joao Lius Marins, Xiaoping Yun, Eric R. An Extended Kalman Filter for Quaternion-Based Orientation Estimation Using MARG Sensors[C]. International Conference on Intelligient Roots and System, 2011(10):2003-2011.

[6] C. D. Mutto, P. Zanuttigh, G. M. Cortelazzo, et al., Scene Segmentation Assisted by Stereo Vision. International Conference on 3D Imaging, Modeling, Processing, Visualization and Transmission, 2011:57 64

[7] L.Y. Cheng, Q. Sun, H. Su1, et al., Design and Implementation of Human-Robot Interactive Demonstration System Based on Kinect. Control and Decision Conference (CCDC), 2012: 971 975

[8] W. Adiprawita, A. R. Ibrahim, Service Oriented Architecture in Robotic as a Platform for Cloud Robotic. International Conference on Cognitive Infocommunications (CogInfoCom), 2012:1 4

[9] Sabatelli S, Sechi F, Fanucci L, et al. A sensor fusion algorithm for an integrated angular position estimation with inertial measurement units[C]. Design, Automation \& Test in Europe Conference \& Exhibition (DATE), 2011. IEEE, 2011: 1-4.

[10] CHEN Hang-ke, ZHANG Dong-sheng, SHENG Xiao-chao, WANG Kai. Research on signal fusion technology of attitude sensor based on Kalman filtering algorithm. [J]. Transducer and Microsystem Technologies,2013,32(12):82-85.

[11] ZHANG Li-jie, CHANG Ji. Attitude and heading measurement system and its data fusion method [J]. Journal of Chinese Inertial Technology, 2011, 19(3): 307-311.

[12] LIU Xian-mei, ZHAO Dan, The literature review of three-dimensional human body motion retrieval technology for content, Computer Engineering and Applications, 2012, 48 (18) : 148 153, 163

[13] LI Hong-Song, LI Da. Some Advancesin Human Motion Analysis, Pattern Recognition and Artificial Intelligence, 2009, 22 (1) : 70 78

[14] D.Alexiadis , P.Daras, P.Kelly et al., Evaluating a Dancer's Performance using Kinect-based Skeleton tracking, Proceedings of the 19th ACM international conference on Multimedia,2011.659 662

[15] Matthew Field, Zengxi Pan, David Stirling et al. Human motion capture sensors and analysis in robotics. Industrial Robot: An International Journal, 2011, 38(2):163-171.

[16] R. Ahmad, M. B. Che Omar, M.S., Yaacob et al. Modeling of Human Motion through Motion Captured Data. IEEE, 2008 